\title{
Severity Prediction System for Real Time Pothole Detection
}

DOI: $10.51201 /$ JUSST/21/07356

\author{
Sumit Bhimte ${ }^{1}$, Hrishikesh hasabnis ${ }^{2}$, Rohit Shirsath ${ }^{3}$, \\ Saurabh Sonar ${ }^{4}$, Mahendra Salunke $^{5}$ \\ ${ }^{1,2,3,4}$ B.E (Computer Engineering), Pimpri Chinchwad College Of Engineering And \\ Research, Ravet, Pune, Maharashtra, India \\ ${ }^{5}$ Asst. Professor, Department of Computer Engineering, Pimpri Chinchwad College Of \\ Engineering And Research, Ravet, Pune, Maharashtra, India,
}

\begin{abstract}
1Email: bhimtesumit118@gmail.com²Email: hrishikeshhasabnis@gmail.com

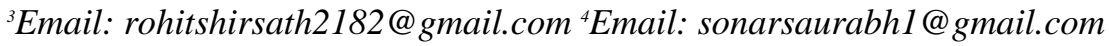

Email: mahendra.salunke@pccoer.in

\begin{abstract}
Pothole Detection System using Image Processing or using Accelerometer is not a new normal. But there is no real time application which utilizes both techniques to provide us with efficient solution. We present a system which can be useful for the drivers to determine the intensity of Pothole using both Image Processing Technology and Accelerometer device-based Algorithm. The challenge in building this system was to efficiently detect a Pothole present in roads, to analyze the severity of Pothole and to provide users with information like Road Quality and best possible route. We have used various algorithms for frequencybased pothole detection. We compared the results.

Apart from that, we selected the best approach suitable for achieving the project goals. We have used a Simple Differentiation-based Edge Detection Algorithm for Image Processing. The system has been built on Map Interfaces for Android devices using Android Studio, which consists of usage of Image Processing Algorithm based Python frameworks which is a sub field of Machine Learning. It is backed by powerful DBMS. This project facilitates use of most efficient technology tools to provide a good user experience, real time application, reliability and improved efficiency.
\end{abstract}

Keywords: Image Processing, Accelerometer, OpenCV, Dark Net, Pothole Detector, Deep Learning.

\section{Introduction}

There are a lot of people who faces severe injuries, traumatic events, accidents, fatal encounters because of poor road facility across different parts of India. This is an attempt to reduce and correct these deadly events. It can also be useful to provide the government with appropriate information. It will also be capable of showing people about different alternatives which can be safer options for travelling. Our attempt is to design a Map based Interface System that takes Real Time Road Images and Pothole Frequency Readings as data using devices like Accelerometer and Camera in order to process the acquired data to check the existence of the pothole and to check the Severity of Potholes and Quality of Road.

In this project we have used cutting-edge technology like YOLOv4 which is a framework developed by Snapchat for real time object detection. Apart from that, we have used 'darknet' library which is extensively developed for computation of 
YOLOv4. This is a supervised machine learning technique where we have manually trained a dataset of 600 images. Also, we have an IOT aspect in our project in which we have used Accelerometer sensor to take the X, Y, Z 3D coordinates. We have applied queries on RDBMS table accordingly to obtain the pothole location in the form of latitude, longitude. The real time camera input will guide the driver about the potential threats in the form of potholes. If any fluctuations are observed even after pothole detection, it is inferred that the pothole is severe. Accordingly, the location of pothole is provided with appropriate color. Else, the pothole is considered to be less risky, which is notified using some different color. This color chromatology is provided on map-based interface application designed for mobile phones. The whereabouts of the project are depicted in Figure 1.

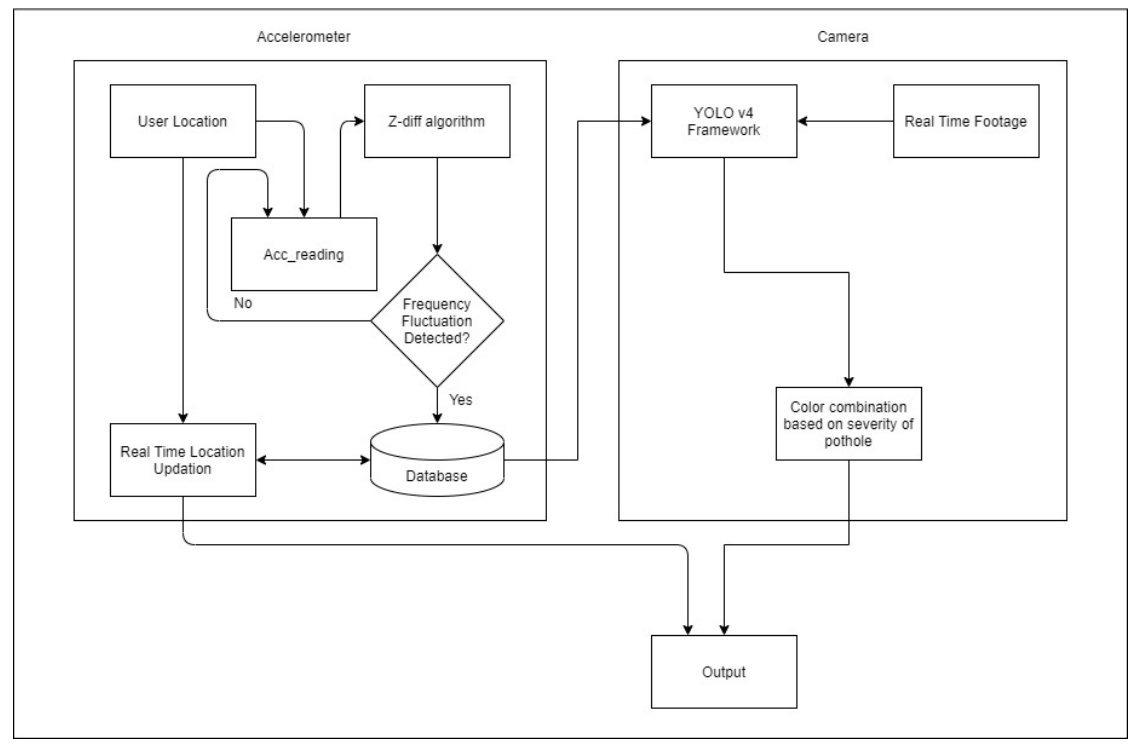

Figure 1. Architecture Diagram for Pothole detection Project

\section{Literature Review}

A Road Quality Detection Method - HUAIJUN WANG, NA HUO, JUNHUAI LI, KAN WANG, AND ZHIXIAO WANG Shaanxi Key, Shaanxi Key Laboratory for Network Computing and Security Technology, School of Computer Science and Engineering, Xi'an University of Technology, Xi'an 710048, China.

In this research paper the accelerometer sensor has fluctuations when the vehicle passes through the remarkably larger. They developed application to gather and manipulate sensor data, and then predict road quality conditions.

Real Time Pothole Detection using Android Smartphones with Accelerometers Artis Ednis,Girts Strazdins, Reinholds Zviedris, Georgijs Kanonirs, Leo Selavo, Digital Signal Processing Laboratory Institute of Electronics and Computer Science.

This paper describes a mobile inbuilt sensing system for road quality detection. Data processing algorithms to processing the accelerometer data are discussed and their processing presented using actual data.

A Modern Pothole Detection technique using Deep Learning - Abhishek Kumar, Chakrapani, Dhruva Jyoti Kavita, Department of Computer Science and Engineering National Institute of Technology, Allahabad

This paper proposed use of YOLOv4, darknet, Computer Vision i.e., OpenCV library as software aspects for image processing to detect potholes. This model is basically based on region-based Convolutional Neural Network (RCNN) and 
darknet. According to the model that has been prepared, accurate video output which shows the presence of pothole is returned for the respective input.

\section{Methodology}

This is a supervised machine learning technique where we have manually trained a dataset of 600 images. Also, we have an IOT aspect in our project in which we have used Accelerometer sensor to take the X, Y, Z 3D coordinates. We have applied queries on RDBMS table accordingly to obtain the pothole location in the form of latitude, longitude. The real time camera input will guide the driver about the potential threats in the form of potholes. If any fluctuations are observed even after pothole detection, it is inferred that the pothole is severe. Accordingly, the location of pothole is provided with appropriate color. Else, the pothole is considered to be less risky, which is notified using some different color. This color chromatology is provided on map-based interface application designed for mobile phones. The following diagram will depict the whereabouts of the project. The proposed methodology is shown in Figure. 2

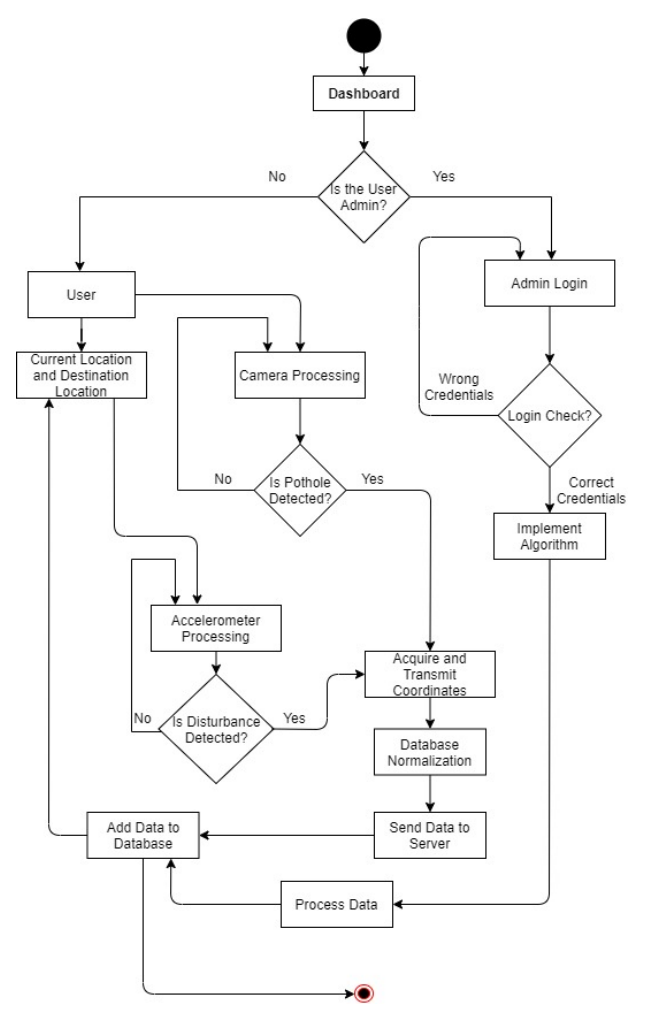

Figure 2. System Architecture

\section{1 : Data Pre-processing}

Data preprocessing is a technique in which we transform the raw data in a useful, clean and efficient format for further training the models. In our module, we transform X, Y, Z coordinate readings obtained from accelerometer into potential pothole locations. Initially it contained 27166 readings upon which stored procedure was applied, which got converted into 8 readings which were the actual pothole locations. For image processing we took a dataset of 600 pothole images which were manually trained by all of us. 


\section{2 : Logic For Accelerometer}

Data in the form of a dataset from the accelerometer sensors were collected on an urban road with various potholes. MansOS based software was used, to obtain raw acceleration data and transmit it through a USB interface to a laptop computer. Consecutive measurements with difference value above specific threshold level represent events. Road Mic pothole detection methodology was used to collect reference data, where Road Mic tests were performed. Research of potential eventrelated features was performed, after the acquisition of the first test data set. The emphases were put on features that do not require resource-intensive signal processing techniques and therefore are suitable for implementation of real-time detection using devices with limited hardware and software resources. It is similar to the z-peak algorithm used in Pothole Patrol and Traffic Sense systems, and is thresholding the acceleration amplitude at Z-axis. The features that classify the measurements are the values exceeding specific thresholds that identify the type of the potholes.

The algorithm is trained in such a way that it assumes about the information regarding the Z-axis position of the accelerometer is known. Additional virtual reorientation of the accelerometer is possible, as described in Neric ell. We used the controlled placement of the accelerometer and eliminated the extra processing required for the virtual reorientation. This also tested on the acquired data set. It searches for the two consecutive measurements with a difference in their values above a specific threshold level was performed, instead of ZTHRESH. Fast changes in vertical acceleration data were detected by the algorithm. It is required by the algorithm to determine the Z-axis position similar to the previous approach. After the analysis of the related work, it was decided by the authors to implement some of the algorithms that could be used for post-processing. The standard deviation of vertical acceleration was one of the techniques which looked promising to implement a resource-constrained device. Pothole detection algorithm STDEV. Measurements with standard deviation value above specific threshold level represent events.

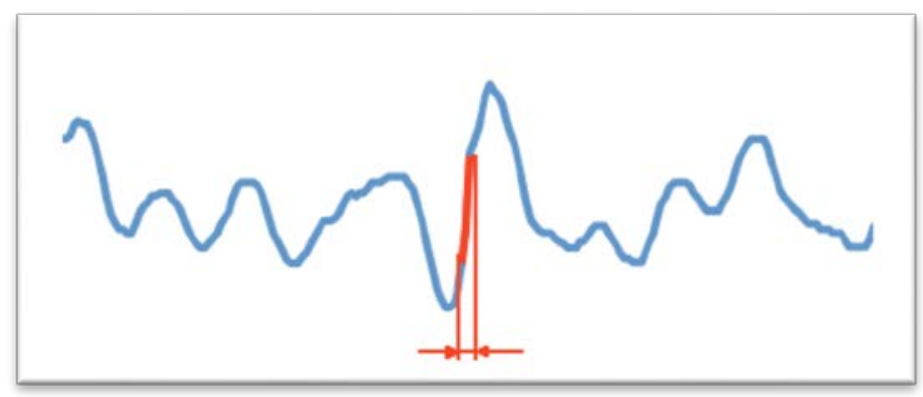

Figure 4. Pothole Detection Algorithm: Z-Diff

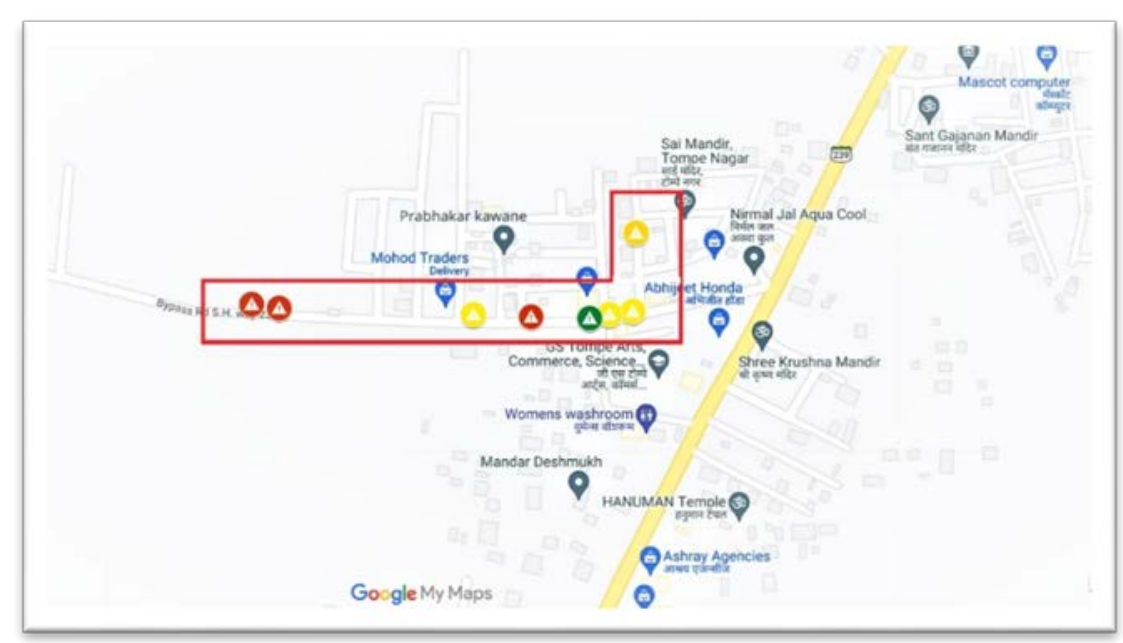


Figure 5. Google Map interface to depict pothole locations

\section{3 : Logic for Image Processing}

We have used high resolution images and videos as input for the purpose of training and building computer vision system. Images are accordingly converted into convenient resolution. We have used YOLOv4, darknet, Computer Vision i.e., OpenCV library as software aspects for this project. We had a dataset consisting of 600 high resolution images, of which 30 images are used for training and remaining images are used for testing. Before providing all the images to create a model, it was necessary to process the images and convert them into suitable format. Initially all the images were in high end resolution which were resized and compressed into suitable processing format but not losing its actual property as well as quality. Also, low light images were enhanced using MIRNET model. We were successful in creating a label called 'Pothole' which was manually provided in each image used for training. Depending on the input images provided it was possible for us to recognize potholes in sample videos.

YOLO framework also known as 'You Only Look Once' deals with object detection. It takes entire image/video in a single instance. It returns boundaries of the detected object in the form of coordinates. It works on the principle of probabilities; higher the obtained probability higher is the accuracy of the detected object. It is a very fast technique which can process 45 frames per second. For training, YOLO takes an image and converts it into $3 \times 3$ grid and checks for the probability of presence of given object (Pothole) in each grid individually. Each grid is assigned values 0 or 1 according to the probability. For testing, images are passed and run-in forward propagation until we obtain output. It uses RCNN family of techniques. According to the model that has been prepared, accurate video output which shows the presence of pothole is returned for the respective input.

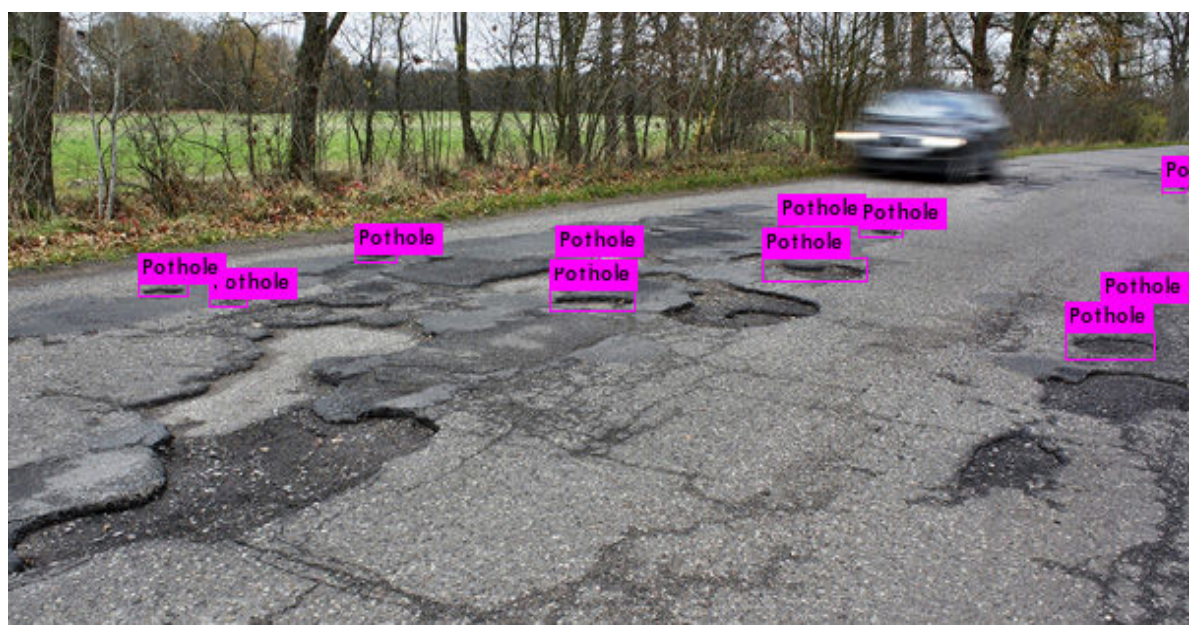

Figure 6. Image Processing Output 


\subsection{Gap Analysis}

\begin{tabular}{|c|c|}
\hline Current Situation & Desired Situation \\
\hline $\begin{array}{c}\text { Pothole detection using only } \\
\text { Accelerometer and only } \\
\text { Image Processing has been } \\
\text { implemented. }\end{array}$ & $\begin{array}{c}\text { To create a system which can use } \\
\text { both Accelerometer as well as } \\
\text { Image Processing to obtain high } \\
\text { accuracy. }\end{array}$ \\
\hline $\begin{array}{c}\text { No technology to predict the } \\
\text { severity of pothole available. }\end{array}$ & $\begin{array}{c}\text { To create an application in such } \\
\text { a way that the severity of pothole } \\
\text { can be predicted using color } \\
\text { chromatology. }\end{array}$ \\
\hline $\begin{array}{c}\text { Dataset for these projects } \\
\text { only work for day light } \\
\text { scenario. }\end{array}$ & $\begin{array}{c}\text { Dataset for our desired project } \\
\text { can work in night light as well. }\end{array}$ \\
\hline $\begin{array}{c}\text { Algorithm like canny edge } \\
\text { has been used in pothole } \\
\text { detection by image } \\
\text { processing. }\end{array}$ & $\begin{array}{c}\text { We will use low light image } \\
\text { enhancement algorithms like } \\
\end{array}$ \\
$\begin{array}{c}\text { MIRNET in order to enhance the } \\
\text { lighting conditions in image and } \\
\text { then use Canny edge algorithm } \\
\text { to achieve image processing for } \\
\text { low light images. }\end{array}$ \\
\hline
\end{tabular}

\section{Conclusion and Future Scope}

We proposed a system where user can get the location of Potholes, the Quality of Road, severity of a pothole, optimal path. The system detects Potholes through Accelerometer and Camera. The frequency related data and the location coordinates are stored in database and respectively processed and updated in real time. The result is shown in the form of Map so that user can choose best possible path and avoid damage or accidents.

Future Scope: In future, we can implement image processing system to avoid pothole in embedded system car. We can implement image processing using thermal imaging, night vision camera to detect potholes in poor lighting conditions. Like Google Maps, we can get information and location about potholes on our smartphones to avoid the accident conditions.

\section{Acknowledgements}

We would like to take this opportunity to thank our internal guide Prof. Mahendra Salunke for giving us all the help and guidance we needed. We are grateful to them for their kind support. Their valuable suggestions were very much helpful. We are also much grateful to Prof. Archana Chaugule, Head of the Computer Engineering Department, Pimpri Chinchwad College of Engineering and Research for her indispensable support as well as suggestions. In the end our special thanks to all the staff members for providing various resources such as a laboratory which has all the needed software platforms and continuous Internet connectivity. 


\section{REFERENCES}

[1] A Road Quality Detection Method - HUAIJUN WANG, NA HUO, JUNHUAI LI, KAN WANG, AND ZHIXIAO WANG Shaanxi Key, Shaanxi Key Laboratory for Network Computing and Security Technology, School of Computer Science and Engineering, Xi'an University of Technology, Xi'an 710048, China

[2] Real Time Pothole Detection using Android Smartphones with Accelerometers - Artis Mednis, Girts Strazdins, Reinholds Zviedris, Georgijs Kanonirs, Leo Selavo, Digital Signal Processing Laboratory

[3] A Modern Pothole Detection technique using Deep Learning - Abhishek Kumar, Chakrapani, Dhruba Jyoti Kalita, Department of Computer Science and Engineering National Institute of Technology,

[4] Deep Learning Approach to Detect Potholes in Real-Time using Smartphone Shebin Silvister, Dheeraj Komandur, Shubham Kokate, Aman Kumar, School of Computer Engineering and Technology Dr. Vishwanath Karad MIT World Peace University India - 2020.

[5] Multi-lane Pothole Detection from Crowd sourced Under sampled Vehicle Sensor Data - Andrew Fox, Member, IEEE, B.V.K. Vijaya Kumar, Fellow,

[6] IEEE, Jinzhu Chen, Member, IEEE, and Fan Bai, Fellow, IEEE 